\title{
ТЕОРИЯ РЕЗОНАНСНОГО КОМБИНАЦИОННОГО РАССЕЯНИЯ ФОТОДИССОЦИИРУЕМЫМИ МОЛЕКУЛАМИ
}

\section{1. Введение}

Целью данной работы является развитие теории комбинационного рассеяния фотодиссоциируемыми молекулами типа $I_{2}$ при возбуждении их в резонансе с полосой поглощения в области фотодиссоциации. Особенность резонансного случая по сравнению с нерезонансным состоит в возникновении помимо рассеяния других компонентов вторичного свечения - люминесценции и горячей люминесценции, причем все они описываются одной формулой - формулой второго порядка по взаимодействию света и вещества для неупругого рассеяния света $\left[{ }^{1-3}\right]$. В связи с этим одной из основных задач теории резонансного рассеяния является решение проблемы классификации вторичного свечения. В $\left[{ }^{1-8}\right]$ была предложена теория резонансного вторичного свечения, позволяющая решить указанную проблему. Эта теория применялась конкретно к примесным центрам кристаллов. Однако основные представления теории и развитые в $\left[{ }^{1-4,8}\right]$ методические приемы не были связаны с конкретной моделью, что делает их полезными и в случае описания резонансного вторичного свечения других объектов. В частности, эти результаты можно непосредственно перенести на стекла, растворы и достаточно плотные пары молекул.

В данной работе результаты этой теории будут использованы для расчета характеристик резонансного комбинационного рассеяния фотодиссоциируемыми молекулами в газе низкого давления. При этом мы не будем рассматривать влияние вращений на спектр резонансного рассеяния. Кроме того, мы ограничимся лишь случаем возбуждения выше энергии диссоциации и не будем учитывать столкновений диссоциированных атомов. В отмеченных предположениях в спектре резонансного вторичного свечения будут иметься релеевское и комбинационное рассеяния, но обычная люминесценция (ОЛ) и горячая люминесценция (ГЛ) будут отсутствовать. Этим резонансное вторичное свечение свободных фотодиссоциируемых молекул отличается от резонансного вторичного свечения достаточно плотного молекулярного газа, растворов и примесных центров кристаллов, где наряду с рассеянием имеются также ГЛ и ОЛ. Столкновения, приводящие к возникновению ОЛ и ГЛ, в данной работе рассматриваться не будут. Отметим, что они практически не влияют на резонансное рассеяние при возбуждении выше фотодиссоциации. Более того, достаточно частые столкновения могут привести к полному исчезновению колебательно-вращательной структуры спектра поглощения и с длинноволновой стороны от 
границы фотодиссоциации. В таком случае полученные ниже формулы для резонансного рассеяния будут справедливы и при возбуждении ниже энергии диссоциации.

Хотя точное аналитическое решение рассматриваемой задачи, возможно, и не существует, удовлетворительное приближенное решение может быть найдено. Это оказывается возможным благодаря наличию в задаче малого параметра $\lambda=\omega_{1} / \sigma$, где $\omega_{1}-$ частота колебаний молекулы в основном электронном состоянии, $\sigma$ - ширина спектра поглощения, что позволяет использовать весьма простое полуклассическое приближение. Это приближение обычно применяется при исследовании влияния эффекта Яна-Теллера на спектр поглощения примесных центров кристаллов $\left[{ }^{9}\right]$, а также в теории резонансного комбинационного рассеяния этих центров $\left[{ }^{8}\right]$. Отметим, что оно близко к хорошо известному в теории спектров поглощения фотодиссоциируемых молекул приближению $\delta$-функции (см. ниже) .

Полученные ниже формулы будут применены к резонансному комбинационному рассеянию молекулы $I_{2}$. В последнее время был опубликован ряд работ, посвященных резонансному комбинационному рассеянию двухатомными молекулами типа $I_{2}$ (см., напр., обзор $\left.\left[{ }^{10}\right]\right), 3$ том числе и такие, в которых получены аналитические формулы для зависимостей амплитуд рассеяния от частоты возбуждения [ $\left.{ }^{11}\right]$. По сравнению с этими работами приведенные ниже формулы являются более общими, поскольку они выведены для произвольного адиабатического потенциала возбужденного состояния. Кроме того, в данной работе впервые получены аналитические формулы, определяющие зависимость сечений рассеяния произвольного порядка как от частоты возбуждения, так и от температуры.

\section{2. Общие формулы}

Рассматриваемое резонансное рассеяние относится к двухфотонным процессам типа

$$
1_{\omega_{0}}+C_{1} \rightarrow 1_{\Omega}+C_{2}
$$

где $1_{\omega_{\jmath}}$ - первичный, а $1_{\Omega}$ - вторичный фотоны, $C_{1}$ и $C_{2}$ - облучаемая светом система соответственно до и после взаимодействия с фотоном. Такие процессы описываются хорошо известной формулой второго порядка $\left[{ }^{12}\right]$ :

$$
I_{\mu \mu^{\prime}}=2 \pi\left|\sum_{\mu^{\prime \prime}} \frac{\left\langle\mu\left|H^{\prime}\right| \mu^{\prime \prime}\right\rangle\left\langle\mu^{\prime \prime}\left|H^{\prime}\right| \mu^{\prime}\right\rangle}{E_{\mu}-E_{\mu^{\prime \prime}}}\right|^{2} \mathrm{Q}_{\mu^{\prime}},
$$

где $I_{\mu \mu^{\prime}}$ - вероятность процесса в единицу времени; начальное $(|\mu\rangle)$, промежуточное $\left(\left|\mu^{\prime \prime}\right\rangle\right)$ и конечное $\left(\left|\mu^{\prime}\right\rangle\right)$ состояния включают состояния электромагнитного поля и вещества; $H^{\prime}$ - гамильтониан их взаимодействия, $E_{\mu}$ и $E_{\mu \prime}$ энергии состояний $|\mu\rangle$ и $\left|\mu^{\prime \prime}\right\rangle, \varrho_{\mu}^{\prime}-$ плотность конечных состояний по шкале энергии. Следует подчеркнуть, что в резонансном случае формула (2) в приведенном виде может оказаться непригодной ввиду возникновения расходимости при обращении в нуль знаменателя. В таком случае необходим учет радиационных поправок. Основной результат этого учета весьма прост: фигурирующие в соотношении (2) энергии получают комплексные добавки [ $\left.{ }^{12}\right]$. Действительные части их имеют смысл радиационной перенормировки энергии уровней, а мнимые - констант радиационного затухания. 
В приближении диполь-дипольного взаимодействия электромагнитного поля и вещества $H^{\prime}=-\vec{d} \cdot \vec{\varepsilon}$, где $\vec{\varepsilon}-$ напряженность электрического поля, $\vec{d}$ - оператор дипольного момента оптических электронов (мы не учитываем ионную поляризуемость, несущественную в оптическом диапазоне). При этом сечение вторичного свечения, испускаемого в телесный угол $d \Theta$ в спектральный интервал $(\Omega, \Omega+d \Omega)$ при возбуждении фотоном частоты $\omega_{0}$, определяется формулой

$$
I\left(\omega_{0}, \Omega\right) d \Omega d \Theta=\sum_{\substack{\alpha \alpha^{\prime} \\ \beta \beta^{\prime}}} i_{\alpha} i_{\alpha^{\prime}} j_{\beta} j_{\beta^{\prime}} W_{\alpha \beta \beta^{\prime} \alpha^{\prime}}\left(\omega_{0}, \Omega\right) d \Omega d \Theta .
$$

Здесь $i_{\alpha}-\alpha$-я компонента вектора $\vec{\varepsilon} / \varepsilon$ поляризации возбуждающего света, $j_{\beta}-\beta$-я компонента вектора поляризации $\vec{j}$ излученного света. Тензор четвертого ранга $W_{\alpha \beta \beta^{\prime} \alpha^{\prime}}\left(\omega_{0}, \Omega\right)$ называется иногда тензором вторичного свечения $\left[{ }^{13,8}\right]$, он имеет вид $\left[{ }^{6}\right]$

$$
W_{\alpha \beta \beta^{\prime} \alpha^{\prime}}\left(\omega_{0}, \Omega\right)=B^{2} \sum_{m} n_{m} \sum_{m^{\prime}}\left(P_{\alpha \beta}\right)_{m m^{\prime}}\left(P_{\beta^{\prime} \alpha^{\prime}}^{+}\right)_{m^{\prime} m} \delta\left(\omega_{0}-\Omega+E_{m}-E_{m^{\prime}}\right),
$$

где $m$ и $m^{\prime}$ - соответственно номера, а $E_{m}$ и $E_{m^{\prime}}-$ энергии начального $|m\rangle$ и конечного $\left\langle m^{\prime}\right\rangle$ состояний вещества, $n_{m}$ - вероятность нахождения вещества в начальном состоянии, $B=2 \pi \Omega^{3 / 2} \omega_{0}^{1 / 2} / c^{2}(c-$ скорость света частоты $\Omega$ и поляризации $\vec{j}$ в среде). Тензор

$$
\left(P_{\alpha \beta}\right)_{m m^{\prime}}=\sum_{m^{\prime \prime}}\left(\frac{\left\langle m\left|d_{\alpha}^{+}\right| m^{\prime \prime}\right\rangle\left\langle m^{\prime \prime}\left|d_{\beta}\right| m^{\prime}\right\rangle}{E_{m}-E_{m^{\prime \prime}}+\omega_{0}-i \gamma^{\prime \prime}}+\frac{\left\langle m\left|d_{\beta}^{+}\right| m^{\prime \prime}\right\rangle\left\langle m^{\prime \prime}\left|d_{\alpha}\right| m^{\prime}\right\rangle}{E_{m}-E_{m^{\prime \prime}}-\omega_{0}}\right)
$$

называется тензором рассеяния [ $\left.{ }^{14}\right]$ или поляризуемостью перехода $m \rightarrow m^{\prime}\left[{ }^{15}\right]\left(\gamma_{m}^{\prime \prime}\right.$ - константа радиационного затухания промежуточного состояния $\left.\left|m^{\prime \prime}\right\rangle\right)$. Первый член в скобках соотношения (5) учитывает процессы, в которых в промежуточном состоянии электромагнитного поля нет фотонов, а второй - процессы с двумя фотонами в промежуточном состоянии поля. Последний член при резонансном возбуждении мал и в дальнейшем не учитывается. В формулах (4) и (5) пренебрегается радиационным затуханием начального $|m\rangle$ и конечного $\left|m^{\prime}\right\rangle$ состояний. В тех случаях, когда этого сделать нельзя, в (5) следует $E_{m}$ заменить на $E_{m}+i \gamma_{m}$, а в (4) $\delta$-функцию - на функцию Лоренца с полушириной $\gamma_{m}+\gamma_{m}$.

Поляризуемость перехода $m \rightarrow m^{\prime}$ можно рассматривать как матричный элемент $\left\langle m\left|P_{\alpha \beta}\right| m^{\prime}\right\rangle$ оператора

$$
P_{\alpha \beta}=i \int_{0}^{\infty} d \tau \mathrm{e}^{-i \omega_{0} \tau-i \tau H} d_{\alpha}^{+} \mathrm{e}^{i \tau \hat{H}} d_{\beta}
$$

который, очевидно, следует назвать оператором поляризуемости, поскольку диагональный матричный элемент $\left\langle m\left|P_{\alpha \beta}\right| m\right\rangle$ совпадает с тензором поляризуемости вещества в состоянии $|m\rangle$, а формула (4) является квантовым аналогом формулы для интенсивности излучения классического осциллятора, совершающего вынужденные колебания. В формуле (6) используются обозначения: $\hat{H}=H+i \gamma$, где $H-$ гамильтониан вещества $\left(H|m\rangle=E_{m}|m\rangle\right)$, а $\gamma$ - коммутируіщий с $H$ оператор радиационного затухания: $\gamma|m\rangle=\gamma_{m}|m\rangle$.

Использование оператора поляризуемости позволяет представить 
тензор вторичного свечения в обычном для теории рассеяния виде [5]

$$
W_{\alpha \beta \beta^{\prime} \alpha^{\prime}}\left(\omega_{0}, \Omega\right)=\frac{B^{2}}{2 \pi} \int_{-\infty}^{\infty} d t \mathrm{e}^{i\left(\omega_{0}-\Omega\right) t}\left\langle P_{\alpha \beta}(t) P_{\left.\beta^{\prime} \alpha^{\prime}\right\rangle}^{+}\right\rangle
$$

Здесь $P_{\alpha \beta}(t)=\exp (i t \hat{H}) P_{\alpha \beta} \exp (-i t \hat{H}),\langle\ldots\rangle=\sum_{m} n_{m}\langle m|\ldots| m\rangle-$ знак

квантовомеханического и статистического усреднений.

Формулы (6) и (7) являются общими. Дальнейшее их упрощение в рассматриваемой задаче возможно, если воспользоваться адиабатическим приближением, согласно которому

$$
|m\rangle=|e\rangle|v\rangle
$$

$(|e\rangle$ - электронное, a $|v\rangle$ - соответствующие ему колебательные состояния; вращений, как отмечалось, мы не учитываем). Если исходное (считаемое основным) электронное состояние $|0\rangle$ достаточно удалено от возбужденных (по сравнению с $k T$ ), то в формуле (7) усреднение можно проводить лишь по колебательным состояниям $|v\rangle$ электронного состояния $|0\rangle$. Учтем также, что в рассматриваемой задаче конечное электронное состояние совпадает с начальным. В таком случае оператор поляризуемости принимает вид

$$
P_{\alpha \beta}=\sum_{e} P_{\alpha \beta}^{e}
$$

где

$$
P_{\alpha \beta}^{e}=i \int_{0}^{\infty} d \tau \exp \left\{-i \omega_{0} \tau-i \tau H_{0}\right\} M_{\alpha, e}^{+} \exp \left\{i \tau H_{e}-\gamma_{e} \tau\right\} M_{\beta, e}
$$

- вклад возбужденного электронного уровня $|e\rangle$ в оператор поляризуемости исходного электронного состояния $|0\rangle$. В соотношении (9) $H_{0}-$ колебательный гамильтониан основного $(|0\rangle)$, а $H_{e}-$ возбужденного $(|e\rangle)$ электронных состояний, $\gamma_{e}-$ коммутирующий с $H_{e}$ оператор радиационного затухания состояния $|e\rangle, M_{\alpha, e}=\left\langle e\left|d_{\alpha}\right| 0\right\rangle-$ электронный матричный элемент дипольного перехода $|0\rangle \rightarrow|e\rangle$.

Интересно отметить, что переменная интегрирования $\tau$ в формуле (9) может быть интерпретирована как время пребывания амплитуды состояния системы в возбужденном электронном состоянии $|e\rangle$. В связи с этим люминесценции соответствует интегрирование в (9) в области больших времен $\tau \sim \bar{\gamma}_{e}^{-1}\left(\bar{\gamma}_{e}^{-1}-\right.$ радиационное время жизни возбужденного электронного состояния $|e\rangle$ при температуре $T$ ), а рассеянию и ГЛ - в области малых времен $\tau\left[{ }^{6,7}\right]$. Последнее обстоятельство позволяет при рассмотрении комбинационного рассеяния не учитывать множитель $e^{-\gamma_{e} \tau}$. (Вопрос об актуальной для рассеяния области времен $\tau$ подробно рассмотрен в $\left[{ }^{7}\right]$; см. также ниже.)

\section{3. Теория поляризуемости в резонансном случае}

3.1. Сравнение с нерезонан сны м случа ем. Известно, что в нерезонансной теории поляризуемости Плачека оператор поляризуемости рассматривается как сходящийся ряд по степеням операторов колебательных координат ६; 


$$
P_{\alpha \beta}=P_{\alpha \beta}^{(0)}+P_{\alpha \beta}^{(1)} \xi+P_{\alpha \beta}^{(2)} \xi^{2} / 2+\ldots,
$$

причем для актуальных значений координат при не слишком высоких температурах первый член гораздо больше второго, второй гораздо больше третьего и т. д., а все коэффициенты в разложении (10) действительны.

В резонансном случае, как это следует из формулы (9), $P_{\alpha \beta}$ является более сложным оператором, зависящим не только от операторов координат, но и от операторов импульсов ядер (напомним, что $H_{e}=T+V_{e}+\Omega_{e}, \quad$ где $\Omega_{e}$ - частота электронного перехода $|0\rangle \rightarrow|e\rangle, V_{e}$ и $T-$ операторы потенциальной и кинетической энергии соответственно). С математической точки зрения именно это обстоятельство приводит к качественному изменению характера спектра вторичного свечения при резонансном возбуждении, выражающемуся в возникновении люминесценции. Но если рассматривается лишь комбинационное рассеяние, то в ряде случаев и при резонансном возбуждении зависимостью $P_{\alpha \beta}$ от операторов импульсов ядер можно пренебречь $\left[{ }^{8}\right]$. Это означает, что теория поляризуемости, основанная на учете зависимости оператора поляризуемости только от колебательных координат, в таких случаях может быть использована и при резонансном возбуждении. Однако здесь, в отличие от нерезонансного возбуждения, оператор поляризуемости не является медленно меняющейся действительной функцией колебательных координат. Вследствие этого резонансное рассеяние приобретает многофононный характер, а мнимые части тензоров комбинационного рассеяния могут даже превышать по величине действительные [ $\left.{ }^{8}\right]$.

В фотодиссоциируемых молекулах типа $I_{2}$ взаимодействие оптических электронов с колебаниями сильное, а спектр поглощения при возбуждении в области фотодиссоциации - сплошной. Поэтому обобщенная (в указанном выше смысле) теория поляризуемости, как мы увидим, применима и здесь.

3.2. Оптическая теорема. Релеевское рассеяние. Для того чтобы выяснить, какие дальнейшие упрощающие предположения относительно оператора поляризуемости можно использовать в исследуемой задаче, рассмотрим вначале релеевское рассеяние. Амплитуда вероятности релеевского рассеяния определяется формулой

$$
\begin{gathered}
a_{i}\left(\omega_{0}\right)=B \sum_{\alpha \beta} i_{\alpha} j_{\beta}\left\langle i\left|P_{\alpha \beta}\right| i\right\rangle= \\
=i B \int_{0}^{\infty} d \tau \mathrm{e}^{-i \omega_{0} \tau}\left\langle i\left|\mathrm{e}^{-i \tau H_{0}} M_{1}^{+} \mathrm{e}^{i \tau H_{e} M_{2}}\right| i\right\rangle,
\end{gathered}
$$

где $M_{1}=\vec{i} \cdot \vec{M}, M_{2}=\vec{j} \cdot \vec{M}$. Разделим ее на действительную и мнимую части. При этом, согласно (11),

$$
\operatorname{Im} a_{i}\left(\omega_{0}\right)=\frac{B}{2} \int_{-\infty}^{\infty} d \tau \mathrm{e}^{-i \omega_{0} \tau}\left\langle i\left|\mathrm{e}^{-i \tau H_{0}} M_{1}^{+} \mathrm{e}^{i \tau H_{e}} M_{2}\right| i\right\rangle,
$$

а действительная часть $a_{i}\left(\omega_{0}\right)$ связана с мнимой дисперсионным соотношением

$$
\operatorname{Re} a_{i}\left(\omega_{0}\right)=\frac{1}{\pi} P \int \frac{\operatorname{Im} a_{i}(x)}{\omega_{0}-x} d x .
$$

(Последнюю формулу можно рассматривать как следствие аналитичности функции $a_{i}\left(\omega_{0}\right)$ относительно $\omega_{0}$.) В случае $M_{2}=M_{1}$ (рассея- 
ние вперед) правая часть формулы (12) с точностью до нормировочного множителя совпадает с обычным выражением для спектра поглощения $\varkappa_{i}\left(\omega_{0}\right)\left[{ }^{2}\right]$ :

$$
\begin{gathered}
\operatorname{Im} a_{i}\left(\omega_{0}\right)=\frac{\omega_{0}}{4 \pi c} x_{i}\left(\omega_{0}\right), \\
\varkappa_{i}\left(\omega_{0}\right)=\frac{4 \pi^{2} \omega_{0}}{c} \int_{-\infty}^{\infty} d \tau \mathrm{e}^{-i \omega_{0} \tau}\langle i| \mathrm{e}^{-i \tau H_{0}} M_{1}^{+} \mathrm{e}^{i \tau H_{e} M_{1}|i\rangle}
\end{gathered}
$$

(Мы учли, что при релеевском рассеянии $\Omega=\omega_{0}$.) Соотношение (13), как известно, является математической формулировкой оптической теоремы $\left[{ }^{16}\right]$. Это соотношение здесь полезно, поскольку оно позволяет перенести приближения, используемые в теории поглощения, на теорию резонансного рассеяния. Ниже будут использованы такие два приближения, близкие между собой: полуклассическое приближение и приближение $\delta$-функции.

3.3. Оператор поляризуемости в полукласси че ском приближении. Если взаимодействие оптических электронов с колебаниями велико, а спектр поглощения бесструктурен, то і формуле (14), а следовательно, и в теории релеевского (и комбинационного) рассеяния можно пренебречь некоммутативностью операторов $H_{0}$ и $V_{e}$ :

$$
\exp \left(i t H_{e}\right)=\exp \left\{i t\left(H_{0}+V+\Omega_{e}\right)\right\} \approx \exp \left\{i t\left(V+\Omega_{e}\right)\right\} \exp \left(i t H_{0}\right) .
$$

Здесь $V=V_{e}-V_{0}-$ разность адиабатических потенциалов возбужденного $\left(V_{e}\right)$ и основного $\left(V_{0}\right)$ электронных состояний (в дальнейшем $\Omega_{e}$ и $V$ выбираются таким образом, чтобы в положении равновесия ядер в основном электронном состоянии $V$ обращалось в нуль). Основания для такого приближения следующие: в Фурье-преобразовании (14) актуальная область интегрирования находится в интервале, удовлетворяющем условию $|\tau| \leqslant \sigma^{-1}$, где $\sigma-$ ширина спектра поглощения. При сильном взаимодействии оптических электронов с колебаниями ширина спектра поглощения гораздо больше частоты колебаний $(\sigma \gg \omega)$. Следовательно, актуальная для поглощения и рассеяния область значений $\tau$ в таком случае значительно меньше периода колебаний: $|\tau| \ll \omega^{-1}$. Но при таких $\tau$ некоммутативностью $H_{0}$ и $V$ в формуле (14) можно пренебречь, в чем легко убедиться, воспользовавшись формулой Фейнмана $\left[{ }^{17}\right]$ :

$$
\mathrm{e}^{i \tau\left(H_{0}+V\right)}=T_{-} \exp \left(i \int_{0}^{\tau} d s V(s)\right) \mathrm{e}^{i \tau H_{0}},
$$

где $V(s)=\exp \left(i s H_{0}\right) V \exp \left(-i s H_{0}\right), T_{-}-$оператор хронологического упорядочения, располагающий операторы в порядке убывания времени справа налево. Действительно, зависимость $V(s)$ от $s$ определяется членами типа ехр $( \pm i \omega s)$. Поскольку $s \leqslant \tau$, то в области $\tau \ll \omega^{-1}$ с точностью до членов $\sim s \omega \sim \omega / \sigma$ можно считать $\exp (i \omega s) \approx 1$. При этом в формуле (16) под интегралом с точностью до малых членов $\omega / \sigma V(s) \approx V$, и (16) сводится к (15).

В полуклассическом приближении (15) и в приближении Кондона $\left(M^{2}=1\right)$ оператор поляризуемости принимает вид

$P_{0}=i \int_{0}^{\infty} d \tau \exp \left\{-i \tau\left(\omega_{0}-\Omega_{e}-V\right)-\varepsilon \tau\right\}=\frac{1}{\omega_{0}-\Omega_{e}-V-i \varepsilon}, \quad \varepsilon \rightarrow 0$. 
Отсюда следует, что при сильном электрон-фононном взаимодействии оператор поляризуемости в первом приближении действительно можно считать зависящим лишь от координат ядер, причем эта зависимость в резонансном случае (т. е. при $\left.\left|\omega_{0}-\Omega_{e}\right| \lesssim \sigma\right)$, в отличие от нерезонансного $\left(\left|\omega_{0}-\Omega_{e}\right| \gg \sigma\right)$, является весьма сильной.

Формула (17) есть нулевое приближение полуклассической теории. Квантовые поправки к этой формуле можно найти, если учесть в (16) в разложении $V(s)$ по $s$ члены $\sim s, s^{2}$ и т. д. Их можно отыскать и другим способом, если воспользоваться формулой

$$
\begin{gathered}
P|i\rangle=i \int_{0}^{\infty} d \tau \mathrm{e}^{-i \omega_{0} \tau+i \tau\left(H_{e}-E_{t}\right)-\gamma_{e} \tau}|i\rangle= \\
=\left(\omega_{0}-H_{e}+E_{i}-i \gamma_{e}\right)^{-1}|i\rangle=\left[E-V-\left(H_{0}-E_{i}\right)\right]^{-1}|i\rangle,
\end{gathered}
$$

где $E=\omega_{0}-\Omega_{e}-i \gamma_{e}$ (в приближении Кондона $\gamma_{e}-c$-число). Эту формулу можно разложить в ряд по степеням $\left(H_{0}-E_{i}\right)\left[{ }^{9}\right]$ :

$$
P|i\rangle=\sum_{n=0}^{\infty} \frac{1}{E-V}\left[\left(H_{0}-E_{i}\right) \frac{1}{E-V}\right]^{n}|i\rangle .
$$

Учитывая, что $E_{i}|i\rangle=H_{0}|i\rangle$, получим для оператора поляризуемости следующий ряд:

$$
P=\sum_{n=0}^{\infty} P_{n}
$$

где

$$
\begin{aligned}
& P_{0}=\frac{1}{E-V} \\
& P_{1}=\frac{1}{E-V}\left[H_{0}, \frac{1}{E-V}\right], \\
& P_{2}=\frac{1}{E-V}\left[H_{0}, \frac{1}{E-V}\left[H_{0}, \frac{1}{E-V}\right]\right]
\end{aligned}
$$

и т. д. $P_{0}$ соответствует полуклассическому приближению, а $P_{1}, P_{2}, \ldots$ дают квантовые поправки к нему.

3.4. Оператор поляризуемости в приближении $\delta$ - функци и. В случае фотодиссоциируемых молекул при расчетах спектра поглощения используется приближение, согласно которому волновая функция относительного движения ядер в диссоциированном состоянии, имеющем энергию $E$, может быть представлена в виде $\delta$-функции:

Тогда

$$
|E\rangle=\delta\left(E-V_{e}\right) .
$$

$$
\begin{aligned}
\mathrm{e}^{i \tau H_{e}} & =\mathrm{e}^{i \tau H_{e}} \sum_{E}|E\rangle\left\langle E\left|=\sum_{E} \mathrm{e}^{i \tau E}\right| E\right\rangle\langle E|= \\
& =\mathrm{e}^{i \tau V_{e}} \sum_{E}|E\rangle\langle E|=\mathrm{e}^{i \tau V_{e} .}
\end{aligned}
$$

В результате оператор поляризуемости в приближении $\delta$-функции примет вид 


$$
P_{\delta}=i \int_{0}^{\infty} d \tau \mathrm{e}^{-i \omega_{0} \tau} \mathrm{e}^{-i \tau H_{0}} \mathrm{e}^{i \tau V_{e}} d \tau
$$

что соответствует полному пренебрежению в формуле (9) оператором кинетической энергии.

Из сравнения формул (22) и (17) следует, что произвольный матричный элемент $\left\langle f\left|P_{\delta}\right| i\right\rangle$ отличается от элемента $\left\langle f\left|P_{0}\right| i\right\rangle$ лишь заменой $V_{e}$ на $V+\Omega_{e}$. В области актуальных координат ядер это различие по порядку величины равно $\omega / \sigma$. Поэтому разница полуклассического и $\delta$-функционального приближений того же порядка, что и квантовая поправка к полуклассическому приближению. Следовательно, полуклассическое и $\delta$-функциональное приближения в случае пренебрежения членами порядка $\omega / \sigma$ совпадают.

Поправки к приближению $\delta$-функции можно найти, если воспользоваться формулой Фейнмана $\left[{ }^{17}\right]$

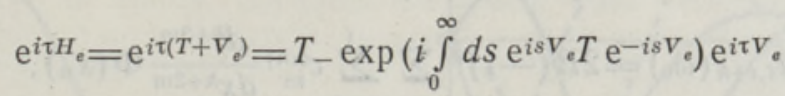

и учесть, разложив упорядоченную экспоненту в ряд по степеням $T$, нужное количество членов. (Член нулевого порядка приводит, очевидно, к приближению $\delta$-функции.)

\section{4. Расчет амплитуд рассеяния для фиксированных начального и конечного состояний (полуклассическое приближение)}

Задачей теории является расчет амплитуды рассеяния $a_{i f}=B\langle i|P| f\rangle$ для всех интересующих эксперимент частот возбуждения $\omega_{0}$, начальных $|i\rangle$ и конечных $|f\rangle$ состояний. В полуклассическом (и в $\delta$-функциональном) приближении соответствующий расчет легко выполнить. Действительно, основываясь на формуле (17) и свойстве сингулярной функции

$$
\lim _{\varepsilon \rightarrow 0} \frac{1}{x-i \varepsilon}=i \pi \delta(x)+P \frac{1}{x}
$$

( $P$ - знак интеграла в смысле главного значения), получим для амплитуды рассеяния в полуклассическом приближении *

$$
\begin{aligned}
& a_{i f}\left(\omega_{0}\right)=B\left\{i \pi \int_{-\infty}^{\infty} d x \psi_{i}(x) \psi_{f}(x) \delta\left[\omega_{0}-V(x)\right]-P \int_{-\infty}^{\infty} d x \frac{\psi_{i}(x) \psi_{f}(x)}{V(x)-\omega_{0}}\right\}= \\
& =B \sum_{n} \frac{1}{\left|V^{\prime}\left(x_{n}\right)\right|}\left\{i \pi \psi_{i}\left(x_{n}\right) \psi_{f}\left(x_{n}\right)-\operatorname{sign} V^{\prime}(x) P \int_{-\infty}^{\infty} d x \frac{\psi_{i}(x) \psi_{f}(x)}{x-x_{n}}\right\} .
\end{aligned}
$$

Здесь использованы соотношения $\delta[f(x)]=\sum_{n} \delta\left(x-x_{n}\right) /\left|f^{\prime}\left(x_{n}\right)\right|$ и $\left[V(x)-\omega_{0}\right]^{-1}=\sum_{n}\left[V^{\prime}\left(x_{n}\right) \cdot\left(x-x_{n}\right)\right]^{-1}$, где $x_{n}{ }^{n}-$ корни уравнения $f(x)=V(x)-\omega_{0}=0 ; V(x)=V+\Omega_{e}$.

Мы видим, что зависимость мнимой части амплитуды комбинационного рассеяния от частоты возбуждения в полуклассическом и $\delta$-функциональном приближениях непосредственно определяется произведе-

\$ Формула (23) справедлива и в приближении $\delta$-функции, если $x_{n}$ определить как корни уравнения $V_{e}(x)-\Omega-E_{i}=0$. 
нием волновых функций начального и конечного колебательных состояний.

Если для колебательных волновых функций основного электронного состояния использовать гармоническое приближение

$$
\psi_{k}=\left[\sqrt{\pi k !} 2^{k}\right]^{-1 / 2} \mathrm{e}^{x^{z} / 2} \frac{d^{k}}{d x^{k}} \mathrm{e}^{-x^{2}}
$$

и учесть формулу

$$
P \int_{-\infty}^{\infty} d x \frac{\mathrm{e}^{-x^{2}}}{y-x}=2 \sqrt{\pi} w(y)
$$

где $w(y)=\exp \left(-y^{2}\right) \int_{0}^{y} d t \exp \left(t^{2}\right)-$ функция Даусона $\left[{ }^{18}\right]$, то для амплитуды рассеяния $k$-го порядка получим

$$
a_{i, i+k}\left(\omega_{0}\right)=2 B(-1)^{k} \sum_{n} \sum_{m=0}^{i} c_{m}^{(i k)} \frac{d^{k+2 m}}{d x_{n}^{k+2 m}} \Phi\left(x_{n}\right),
$$

где

$$
\begin{aligned}
& \Phi\left(x_{n}\right)=\frac{1}{\left|V^{\prime}\left(x_{n}\right)\right|}\left[i \frac{\sqrt{\pi}}{2} \mathrm{e}^{\left.-x_{n}^{2}+\operatorname{sign} V^{\prime}\left(x_{n}\right) w\left(x_{n}\right)\right]}\right. \\
& c_{m}^{(i k)}=\frac{\sqrt{i !(i+k) !}}{2^{(k+2 m) / 2} m !(i-m) !(k+m) !} .
\end{aligned}
$$

Аналогичная формула для амплитуды рассеяния, но только в линейном для адиабатического потенциала $V$ приближении, была получена в $\left[{ }^{11}\right]$. Подчеркнем, что здесь формула (25) получена для произвольного вида $V$.

Выпишем в явном виде полученные зависимости от частоты возбуждения для первых обертонов.

a) Рассеяние первого порядка с нижайших уровней $(i=0,1)$ :

$$
\begin{aligned}
& a_{01}(x) \sim \sqrt{2}\{1-2 x F(x)\}, \\
& a_{12}(x) \sim 2\left\{x^{2}-\left(2 x^{3}-x\right) F(x)\right\},
\end{aligned}
$$

где

$$
F(x)=i \frac{\sqrt{\pi}}{2} \mathrm{e}^{-x^{2}}+w(x) .
$$

б) Рассеяние второго порядка:

$$
\begin{aligned}
& a_{02}(x) \sim \sqrt{2}\left\{-x+\left(2 x^{2}-1\right) F(x)\right\} \\
& a_{13}(x) \sim \frac{4}{\sqrt{6}}\left\{-\left(x^{3}-x\right)+\left(2 x^{4}-3 x^{2}\right) F(x)\right\} .
\end{aligned}
$$

в) Рассеяние третьего порядка:

$$
a_{03}(x) \sim \frac{2}{\sqrt{3}}\left\{x^{2}-1+\left(2 x^{3}-3 x\right) F(x)\right\}
$$


Теория резонансного комбинационного рассеяния...

45

$$
a_{14}(x) \sim \frac{1}{\sqrt{3}}\left\{2 x^{4}-5 x^{2}+\left(4 x^{5}-12 x^{3}+3 x\right) F(x)\right\}
$$

Здесь $x=x\left(\omega_{0}\right)$ - функция частоты возбуждения, определяемая из решения уравнения $V(x)-\omega_{0}=0$. (В (28) предполагается, что это уравиение имеет один корень; $x_{1}=x$. Если это не так, то в этих формулах следует $x$ заменить на $x_{n}$ и добавить сумму по $n$.) Отметим, что в линейном для $V(x)$ приближении $V(x)=V_{0}+b x, x-$ линейная функция $\omega_{0}: x=\left(\omega_{0}-V_{0}\right) / b$.

Рассчитанные на основе (28) действительные и мнимые части амплитуд показаны на рисунке.
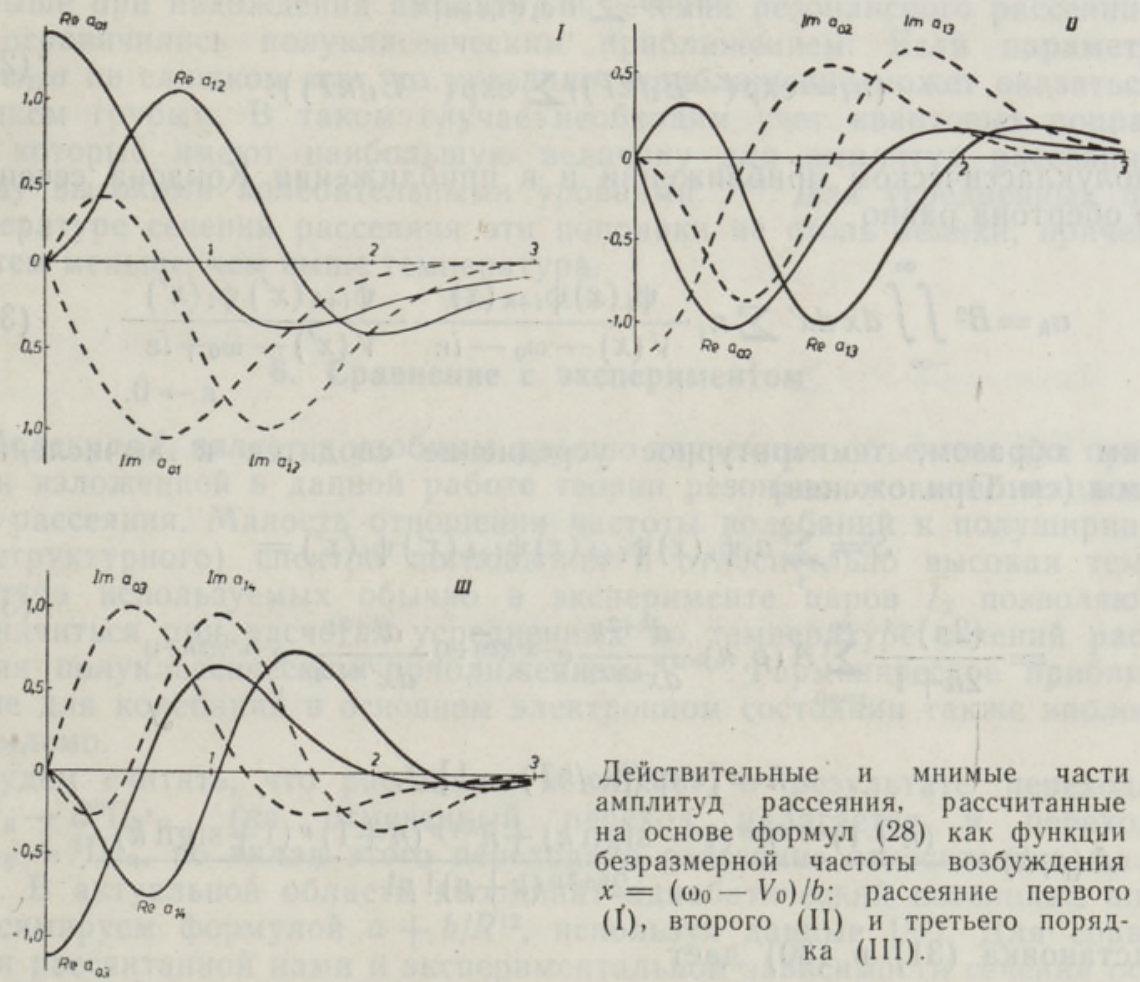

Действительные и мнимые части амплитуд рассеяния, рассчитанные на основе формул (28) как функции безразмерной частоты возбуждения $x=\left(\omega_{0}-V_{0}\right) / b:$ рассеяние первого (I), второго (II) и третьего порядка (III).

Отметим, что в эксперименте непосредственно измеряются не амплитуды, а сечения рассеяния. Тем не менее знание зависимостей амплитуд рассеяния от $\omega_{0}$ важно, поскольку, как правило, при рассмотрении рассеяния существен учет как резонансного, так и нерезонансного уровней. Причем в первом приближении вклад этих уровней в амплитуды рассеяния аддитивен. ${ }^{* *}$ Поэтому сечение рассеяния $i \rightarrow i+k$ определяется формулой

$$
\sigma_{i, i+h}\left(\omega_{0}\right)=\left|a_{i, i+k}\left(\omega_{0}\right)+a_{i, i+k}\left(\omega_{0}\right)\right|^{2},
$$

где $a^{\text {нр }}$ учитывает вклад в амплитуду рассеяния нерезонансных уров-

В тех случаях, когда электронные уровни вырождены (квазивырождены), это не так; здесь существен эффект Яна-Теллера, приводящий к колебательному «перепутыванию» уровней. Теория резонансного комбинационного рассеяния с учетом эффекта Яна-Теллера была развита в $\left[{ }^{8}\right]$. 
ней. Отметим также, что экспериментально выделить рассеяние $i \rightarrow i+k$ от рассеяний того же порядка $k$, но других исходных уровней $i$, можно лишь в том случае, когда колебательные уровни $i$ в достаточной степени неэквидистантны.

\section{5. Усредненные по температуре сечения рассеяния}

Во многих случаях в эксперименте линии рассеяния $i \rightarrow i+k$ для разных $i$, но одинаковых $k$ не разрешаются. Тогда для сравнения теории с экспериментом необходимо вычислить сечения комбинационного рассеяния, усредненные по температуре

$$
\begin{gathered}
\sigma_{k}=\sum_{i} n_{i}\left|a_{i, i+k}\right|^{2} \\
\left(n_{i}=\exp \left(-E_{i} / k T\right) / \sum_{i} \exp \left(-E_{i} / k T\right)\right) .
\end{gathered}
$$

В полуклассическом приближении и в приближении Кондона сечение $k$-го обертона равно

$$
\sigma_{k}=B^{2} \int_{-\infty}^{\infty} \int_{-\infty} d x d x^{\prime} \sum_{i} n_{i} \frac{\psi_{i}(x) \psi_{i+k}(x)}{V(x)-\omega_{0}-i \varepsilon} \cdot \frac{\psi_{i+k}\left(x^{\prime}\right) \psi_{i}\left(x^{\prime}\right)}{V\left(x^{\prime}\right)-\omega_{0}+i \varepsilon},
$$

Таким образом, температурное усреднение сводится к вычислению суммы (см. Приложение)

$$
\begin{gathered}
S=\sum_{i} n_{i} \psi_{i}(x) \psi_{i+k}(x) \psi_{i+k}\left(x^{\prime}\right) \psi_{i}\left(x^{\prime}\right)= \\
=\frac{(2 \pi)^{-1}}{2 \bar{n}+1} \sum_{p=0}^{\infty} A(p, k) \frac{d^{k+2 p}}{d x^{k+2 p}} \mathrm{e}^{-x^{2} /(2 \bar{n}+1)} \frac{d^{k+2 p}}{d x^{\prime k+2 p}} \mathrm{e}^{-x^{\prime 2 /(2 \bar{n}+1)},}
\end{gathered}
$$

где

$$
\begin{gathered}
\bar{n}=[\exp (\bar{n} \omega / k T)-1]^{-1}, \\
A(p, k)=\frac{(\bar{n}+1)^{k+p} \bar{n}^{p}(1-\operatorname{sign} k)+\bar{n}^{k+p}(\bar{n}+1)^{p}(1+\operatorname{sign} k)}{2^{k+2 p}(k+p) ! p !} .
\end{gathered}
$$

Подстановка (31) в (30) дает

$$
\sigma_{k}=\frac{B^{2} / 2 \pi}{2 \bar{n}+1} \sum_{p=0}^{\infty} A(p, k)\left|\int_{-\infty}^{\infty} d x \frac{\frac{d^{k+2 p}}{d x^{k+2 p}} \mathrm{e}^{\left.-x^{2} / 2 \bar{n}+1\right)}}{V(x)-\omega_{0}+i \varepsilon}\right|_{\varepsilon \rightarrow 0}^{2} .
$$

Используя те же соотношения, что и в предыдущем разделе, легко убеждаемся, что интеграл в формуле (32) равен производной $(k+2 p)$-го порядка уже известной фунции Ф $(x)$ (см. (26)):

$$
\sigma_{k}=2 B^{2} \sum_{p=0}^{\infty} \frac{A(p, k)}{(2 \bar{n}+1)^{k+2 p}}\left|\sum_{n} \Phi_{k+2 p}\left(\tilde{x}_{n}\right)\right|^{2},
$$

где

$\Phi_{m}\left(\tilde{x}_{n}\right)=\frac{d^{m}}{d \tilde{x}_{n}^{m}} \Phi\left(\tilde{x}_{n}\right)=\frac{d^{m}}{d \tilde{x}_{n}^{m}}\left\{\frac{1}{\left|V^{\prime}\left(\tilde{x}_{n}\right)\right|}\left[i \frac{\sqrt{\pi}}{2} \mathrm{e}^{-\tilde{x}_{n}^{2}}+\operatorname{sign} V^{\prime}\left(\tilde{x}_{n}\right) w\left(\tilde{x}_{n}\right)\right]\right\}$, 
$\tilde{x}_{n}=x_{n} /(2 \bar{n}+1)$, а $x_{n}-$ корень уравнения $V(x)-\omega_{0}=0$. Запишем в явном виде формулы для сечения стоксовых $\left(\sigma^{S}\right)$ и антистоксовых $\left(\sigma^{A}\right)$ линий комбинационного рассеяния $k$-го порядка

$$
\begin{aligned}
\sigma_{k}^{S} & =2 B^{2} \sum_{p=0}^{\infty} \frac{(\bar{n}+1)^{k+p} \bar{n}^{p}}{[2(2 \bar{n}+1)]^{k+2 p}(k+p) ! p !}\left|\sum_{n} \Phi_{k+2 p}\left(\tilde{x}_{n}\right)\right|^{2}, \\
\sigma_{k}^{A} & =2 B^{2} \sum_{p=0}^{\infty} \frac{\bar{n}^{k+p}(\bar{n}+1)^{p}}{[2(2 \bar{n}+1)]^{k+2 p}(k+p) ! p !}\left|\sum_{n} \Phi_{k+2 p}\left(\tilde{x}_{n}\right)\right|^{2} .
\end{aligned}
$$

Эти формулы в линейном для $V$ приближении были получены ранее в $[19,20]$.

Выше при нахождении амплитуд и сечений резонансного рассеяния мы ограничились полуклассическим приближением. Если параметр $\lambda=\omega_{1} / \sigma$ не слишком мал, то указанное приближение может оказаться слишком грубым. В таком случае необходим учет квантовых поправок, которые имеют наибольшую величину для амплитуд рассеяния между высокими колебательными уровнями. *** Для усредненных по температуре сечений рассеяния эти поправки не столь велики, причем они тем меньше, чем выше температура.

\section{6. Сравнение с экспериментом}

Молекула $I_{2}$ является удобным, хорошо изученным объектом для проверки изложенной в данной работе теории резонансного комбинационного рассеяния. Малость отношения частоты колебаний к полуширине (бесструктурного) спектра поглощения и относительно высокая температура используемых обычно в эксперименте паров $I_{2}$ позволяют ограничиться при расчетах усредненных по температуре сечений рассеяния полуклассическим приближением. **** Гармоническое приближение для колебаний в основном электронном состоянии также вполне приемлемо.

Будем считать, что рассеяние возникает в результате перехода $X^{1} \Sigma_{0^{*}} \rightarrow B^{3} \Pi_{0^{*}} u$ (на отмеченный переход налагается и переход $X^{1} \Sigma_{0^{+}} \rightarrow{ }^{1} \Pi_{1 u}$, но вклад этого перехода в рассеяние относительно мал $\left.\left[{ }^{21}\right]\right)$. В актуальной области координат адиабатический потенциал аппроксимируем формулой $a+b / R^{12}$, используя данные $\left.{ }^{21}\right]$. Для сравнения рассчитанной нами и экспериментальной зависимости сечения основной стоксовой линии комбинационного рассеяния от частоты возбуждающего света используем данные $\left[{ }^{22}\right]$. Результаты сопоставления приведены в табл. 1.

Таблица 1

\begin{tabular}{lccccccc}
\hline$\lambda(\AA)$ & 4965 & 4880 & 4765 & 4727 & 4655 & 4579 & 4545 \\
\hline$\sigma_{\text {теор }}$ & 1,22 & 1 & 0,65 & 0,5 & 0,29 & 0,15 & 0,12 \\
\hline$\sigma_{\text {экеп }}$ & 1,20 & 1 & 0,61 & 0,48 & 0,33 & 0,30 & 0,28 \\
\hline
\end{tabular}

\footnotetext{
*** Это связано с тем, что квантовые 'поправки определяются операторами $d / d x$, вследствие чего они тем больше, чем больше узлов имеет функция $\psi_{i}(x) \psi_{f}(x)$. **** Относительная ошибка не превышает $10 \%$. Отметим, что для амплитуд $a_{i f}$ при $i, f \geqslant 3$ относительная ошибка достигает $50 \%$ и более.
} 
В табл. 2 приведены полученные нами результаты относительно высших обертонов и соответствующие экспериментальные данные $\left[{ }^{23}\right]$. Расчеты сделаны для 400 (температура проведения эксперимента) и $600 \mathrm{~K}$ (результаты в скобках). При расчетах в сумме формулы (34) учтены только первые, наиболее важные члены.

таблица 2

\begin{tabular}{cccccc}
\hline$k$ & 1 & 2 & 3 & 4 \\
\hline$\left(\sigma_{k} / \sigma_{1}\right)_{\text {теор }}$ & $1(1)$ & $0,77(0,73)$ & $0,56(0,47)$ & $0,48(0,35)$ \\
\hline$\left(\sigma_{k} / \sigma_{1}\right)_{\text {зкеп }}$ & 1 & 0,71 & 0,55 & 0,45 \\
\hline
\end{tabular}

Отсюда, в частности, следует, что отношения сечений резонансного рассеяния высших порядков к низшим при повышении температуры уменьшаются. В случае нерезонансного комбинационного рассеяния температурная зависимость отмеченных отношений имеет, как известно, противоположную картину.

\section{Приложение}

Вычисление $S=\sum_{i} n_{i} \psi_{i}(x) \psi_{i+h}(x) \psi_{i+k}\left(x^{\prime}\right) \psi_{i}\left(x^{\prime}\right)$.

Перепишем вначале сумму $S$ в виде

$$
S=\int_{-\infty}^{\infty} d \xi d \xi^{\prime} \sum_{i} n_{i} \psi_{i}(\xi) \delta(x-\xi) \psi_{i+k}(\xi) \psi_{i+k}\left(\xi^{\prime}\right) \delta\left(x^{\prime}-\xi^{\prime}\right) \psi_{i}\left(\xi^{\prime}\right) .
$$

Используем затем интегральное представление $\delta$-функций:

$$
\begin{gathered}
S=(2 \pi)^{-2} \int_{-\infty}^{\infty} d \tau d \tau^{\prime} \sum_{i} n_{i}\langle i|\exp \{i(x-\xi) \tau\}| i+k\rangle \times \\
\times\left\langle i+k\left|\exp \left\{-i\left(x^{\prime}-\xi^{\prime}\right) \tau^{\prime}\right\}\right| i\right\rangle .
\end{gathered}
$$

Чтобы просуммировать по «промежуточным» состояниям $i+k$, введем еще одну $\delta$-функцию

$$
\begin{gathered}
S=(2 \pi)^{-2} \int_{-\infty}^{\infty} d \tau d \tau^{\prime} \exp \left(i x \tau-i x^{\prime} \tau^{\prime}\right) \sum_{i, m} n_{i} \int_{-\varepsilon}^{\varepsilon} d y \delta\left(y+E_{i}-E_{m}+\omega k\right) \times \\
\times\langle i|\exp (-i \xi \tau)| m\rangle\left\langle m\left|\exp \left(i \xi^{\prime} \tau^{\prime}\right)\right| i\right\rangle, \quad \varepsilon \rightarrow 0 .
\end{gathered}
$$

Тогда, использовав интегральное представление этой $\delta$-функции, получим

$$
\begin{gathered}
S=(2 \pi)^{-3} \int_{-\infty}^{\infty} d \tau d \tau^{\prime} \exp \left(i x \tau-i x^{\prime} \tau^{\prime}\right) \int_{-\varepsilon}^{\varepsilon} d y \int_{-\infty}^{-\infty} d t \exp \{-i(y+\omega k) t\} \times \\
X\left\langle\mathrm{e}^{-i \xi \tau} \mathrm{e}^{i \xi(t) \tau^{\prime}}\right\rangle,
\end{gathered}
$$

где $\xi(t)=\exp \left(i t H_{0}\right) \xi \exp \left(-i t H_{0}\right)-$ координата $\xi$ в представлении Гейзенберга.

Для нахождения коррелятора в (П3) воспользуемся теоремой Блоха о парных корреляторах 


$$
\left\langle\mathrm{e}^{-i \xi \tau} \mathrm{e}^{i \xi(t) \tau^{\prime}}\right\rangle=\sum_{m=0}^{\infty} \frac{\langle\xi \xi(t)\rangle^{m}}{m !}\left(\tau \tau^{\prime}\right)^{m} \exp \left\{-\frac{\tau^{2}+\tau^{\prime 2}}{2}\left\langle\xi^{2}\right\rangle\right\},
$$

где

$$
\begin{aligned}
\langle\xi \xi(t)\rangle= & \frac{1}{2}\left[(\bar{n}+1) \mathrm{e}^{i \omega t}+\bar{n} \mathrm{e}^{-i \omega t}\right], \\
& \left\langle\xi^{2}\right\rangle=(2 \bar{n}+1) / 2, \\
\bar{n}= & {[\exp (\bar{n} \omega / k T)-1]^{-1} . }
\end{aligned}
$$

Представив в (П4)

$$
\langle\xi \xi(t)\rangle^{m}=\sum_{p=0}^{m} \mathrm{e}^{i t \omega(m-2 p)} \frac{(\bar{n}+1)^{m-p} \bar{n}^{p} m !}{(m-p) ! p ! 2^{m}},
$$

можем в (П3) проинтегрировать по $t$. При этом мы получим $\delta(y+\omega k-\omega(m-2 p))$. Учитывая, что

$$
\int_{-\varepsilon}^{\varepsilon} d y \delta[y+\omega k-\omega(m-2 p)]_{\mid \varepsilon \rightarrow 0}=\left\{\begin{array}{lll}
1 & \text { при } k=m-2 p ; \\
0 & \text { при } k \neq m-2 p,
\end{array}\right.
$$

получим окончательно

$$
\begin{gathered}
S=\frac{1}{8 \pi^{2}} \int_{-\infty}^{\infty} \int d \tau d \tau^{\prime} \exp \left(i x \tau-i x^{\prime} \tau^{\prime}\right) \sum_{p=0}^{\infty} A(p, k) \cdot\left(\tau \tau^{\prime}\right)^{k+2 p} \times \\
\times \exp \left\{-\frac{\tau^{2}+\tau^{\prime 2}}{4}(2 \bar{n}+1)\right\}= \\
=\frac{(2 \pi)^{-1}}{2 \bar{n}+1} \sum_{p=0}^{\infty} A(p, k) \frac{d^{k+2 p}}{d x^{k+2 p}} \mathrm{e}^{-x^{2} /(2 \bar{n}+1)} \frac{d^{k+2 p}}{d x^{\prime k+2 p}} \mathrm{e}^{-x^{\prime 2} /(2 \bar{n}+1)},
\end{gathered}
$$

где

$$
A(p, k)=\frac{(\bar{n}+1)^{k+p} \bar{n}^{p}(1-\operatorname{sign} k)+\bar{n}^{k+p}(\bar{n}+1)^{p}(1+\operatorname{sign} k)}{2^{2(k+2 p)}(k+p) ! p !} .
$$

\section{Л И Т Р Р Т У Р А}

1. Техвер И., Хи жняков В., Изв. АН ЭССР, Сер. физ.-матем. и техн. наук. 15,9 (1966).

2. Hizhnyakov, V., Tehver, I., Phys. stat. sol., 21, 755 (1967).

3. Hizhnyakov, V., Rebane, K., Tehver, I., In: Light Scattering Spectra of Solids, ed. G. B. Wright, Springer-Verlag, New York, 1969, p. 513.

4. Hizhnyakov, V., Tehver, I., Phys. stat. sol., 39, 67 (1970).

5. Х и жняков В., Р еб ан е К., Т ехвер И., Изв. АН ЭССР, Сер. физ.-матем. и техн. наук, 16, 207 (1967).

6. Р еб ан е К. К., Хи жняков В. В., Вторичное свечение примесного центра - люминесценция, горячая люминесценция и рассеяние. Препринт ИФА-28, Тарту, 1973.

7. Rebane, K. K., T ehver, I. J., Hizhnyakov, V. V., In: Proc. 1st SovietAmerican Symposium, eds. V. M. Agranovich, J. L. Birman, M., 1976, p. 467.

8. Hizhnyakov, V., Tehver, I., In: Proc. 2nd Intern. Conf., ed. M. Balkanski, Paris, 1971, p. 57.

9. Loorits, V., Hizh n y kov, V., In: Physics of Impurity Centres in Crystals, ed. G. S. Zavt, Tallinn, 1972, p. 453.

10. Rousseau, D. L., Willi a m s, P. F., J. Chem. Phys., 64, 3519 (1976).

11. Van Labeke, D., Ja con, M., Berjot, M., B ernard, L., J. Raman Spectrosc., $2,219(1974)$. 
12. Г а й т ле р В., Квантовая теория излучения, M., 1956.

13. Hizhnyakov, V., Tehver, I., In: Physics of Impurity Centres in Crystals, ed. G. S. Zavt, Tallinn, 1972, p. 607.

14. Бр андмюллер И., Мозер Г., Введение в спектроскопию комбинационного рассеяния света, М., 1964.

15. Борн М., Хуан Кунь, Динамическая теория кристаллических решеток, М., 1958.

16. Л ан д а у Л. Д., Л и фш и ц Е. М., Квантовая механика, М., 1963.

17. F e y n m a n, R. P., Phys. Rev., 84, 108 (1951).

18. К а рпов К. А., Таблицы функции $w(z)=e^{-z^{2}} \int_{0}^{z} d x e^{x^{2}}$ в комплексной области, М., 1954.

19. Техв е р И. Ю., Тр. ИФА АН ЭССР, № 32, 31 (1967).

20. Т ехвер И. Ю., Некоторые вопросы теории комбинационного рассеяния примесными центрами кристаллов, Канд. дис., Тарту, 1968.

21. Telli ing hu is e n, J., J. Chem. Phys., 58, 2821 (1973).

22. Ber jot, M., Jacon M., Bernard, L., Opt. Commun., 4, 117 (1971).

23. Holzer, W., Murphy, W. F., Bernstein, H. J., J. Chem. Phys., 52, 399 (1970).

$\begin{array}{cc}\text { Институт физики } & \text { Поступила в редакцию } \\ \text { Академии наук Эстонской } & \text { CCP } \\ 12 / \mathrm{I} & 1977\end{array}$

V. HIŽNJAKOV, F. SOKOLOV, Imbi TEHVER

\section{FOTODISSOTSIEERUVATE MOLEKULIDE RESONANTSE KOMBINATSIOONHAJUMISE TEOORIA}

On esitatud tecoria, mis käsitieb valguse kombinatsioonhajumist $I_{2}$-tüüpi molekulidel, kui viimaseid ergastada fotodissotsiatsiooni piirkonda. On tuletatud hajumise amplituudi üldine valem, konkretiseerimata ergastatud elektronseisundi potentsiaalkõvera kuju. Ohtlasi on analüütiliselt leitud meelevaldset järku kombinatsioonhajumise ristlôige kui ergastussageduse funktsioon ning võrreldud seda $I_{2}$-molekulil teostatud eksperimendi andmetega.

\section{HIZHNYAKOV, F. SOKOLOV, Imbi TEHVER}

\section{THEORY OF RESONANT RAMAN SCATTERING ON PHOTODISSOCIATING MOLECULES}

The resonant Raman scattering on molecules of $I_{2}$ type excited above the photodissociation limit is examined theoretically. The expression of scattering amplitude is analytically found for an arbitrary potential curve of the excited electronic state. The thermally averaged Raman cross-sections as functions of the excitation frequency are also calculated and compared with the experimental data on $I_{2}$ molecule. 